\title{
Endovascular Treatment of Ruptured Dissecting Posterior Inferior Cerebellar Artery Aneurysms
}

\author{
I. IOANNIDIS, N. NASIS, A. ANDREOU \\ Department of Neurosurgery and Interventional Neuroradiology, "HYGEIA” Hospital; Athens, Greece
}

Key words: posterior inferior cerebellar artery, dissecting aneurysms, embolization

\section{Summary}

Dissecting aneurysms of the posterior inferior cerebellar artery (PICA) distal to its origin from vertebral artery (VA) are very rare. Although rare, they associated with a high risk of rebleeding and they present a therapeutic challenge. This study reviewed the clinical presentations, angiographic characteristics of dissecting aneurysms of the PICA and to assess the clinical and angiographic outcomes of patients who underwent endovascular treatment.

Ten patients with ten dissecting aneurysms who underwent endovascular treatment were identified in the clinical records of a single medical center from January 2000 to December 2010. The mean follow-up duration was 2.8 years.

All patients presented with subarachnoid hemorrhage (SAH). They all underwent endovascular treatment, which included occlusion of the dissected segment and the parent artery after detailed angiographic evaluation of the vascular anatomy, and test occlusion of the PICA. In all patients the endovascular treatment was successfully completed without procedure related complications. Long-term follow-up studies in seven out of ten patients showed complete occlusion of the aneurysm with no new neurologic deficits. The clinical outcome was good in eight cases, whereas two patients with poor clinical condition at admission died during their initial hospital stay.

Endovascular occlusion of the parent vessel and the dissected segment is relatively safe treatment option for dissecting aneurysms of the PICA distal to its origin.

\section{Introduction}

Aneurysms of the posterior inferior cerebellar artery (PICA) are uncommon, accounting for $0.5 \%-3 \%$ of all intracranial aneurysms ${ }^{1}$. Most lesions arise near the origin of the PICA from the vertebral artery (VA).

A much smaller proportion, termed distal PICA aneurysms, arise from more peripheral PICA segments. These aneurysms are also classified into saccular and fusiform types. Saccular lesions are hemodynamically generated lesions that usually arise at bifurcations or vessel bends, and can be treated either surgically or endovascularly without sacrifice of the parent vessel.

Fusiform aneurysms arise spontaneously anywhere in the course of the vessel as a consequence of dissection.

Dissecting aneurysms of the PICA are very rare lesions accounting for only 0.5 to $0.7 \%$ of all intracranial aneurysms ${ }^{2}$ and their treatment is controversial. The artery enters proximally and exits distally from the affected vessel segment and therefore cannot be treated without parent vessel occlusion. Surgical procedures carry a high risk of neurologic complications due to the proximity of the vessel to brain stem and lower cranial nerves. Endovascular treatment has been proposed as an alternative to surgery.

The present study retrospectively reviewed a consecutive series of ten patients with ruptured dissecting PICA aneurysms treated endovascularly with parent vessel occlusion in a single center. 


\section{Materials and Methods}

We retrospectively reviewed the records of all patients with intracranial aneurysms treated at our referral center between January 2000 and August 2010. Of 935 patients with 1067 intracranial aneurysms treated during this period, we identified ten patients $(1.07 \%)$ with ten dissecting aneurysms of the PICA distal to its origin from the VA. Dissecting aneurysm was diagnosed if an irregular fusiform dilatation with or without associated narrowing of the PICA was demonstrated angiographically. Patients with dissecting aneurysms involving the vertebral arteries, fusiform PICA aneurysms associated with arteriovenous malformations and nondissecting (saccular aneurysms located at bifurcations or vessel bends) distal PICA aneurysms were excluded. Hospital records, preand post-operative neuroimaging studies, and operative reports were reviewed. Follow-up studies ranged from six months to five years. Only one patient was lost to follow-up review.

The mean age of the ten patients (seven women, three men) was 58.2 years (range, 54.078.0 years). All patients presented with SAH and pretreatment clinical status was recorded from the patient files according to the Hunt \& Hess classification ${ }^{3}$. All patients underwent four-vessel cerebral angiography and a dissecting PICA aneurysm was diagnosed as the cause of bleeding.

According to the anatomical classification described by Lister et al. ${ }^{4}$ the location of the aneurysm was recorded as anterior medullary, lateral medullary, tonsillomedullary, telovelotonsillar, or cortical. The presence and size of potential collateral vessels (contralateral PICA, ipsilateral anterior inferior cerebellar artery, and superior cerebellar artery) were evaluated angiographically. Test occlusion before permanent PICA occlusion was performed by temporary inflation of a balloon (HyperForm Occlusion Balloon System, ev3) in the vertebral artery across the origin of the PICA with simultaneous contrast injection into the contralateral vertebral artery in all cases. Evaluation of leptomeningeal collaterals was based on the extent of retrograde contrast opacification of distal PICA branches on delayed angiographic images. If the collaterals reconstituted the segment adjacent to the occluded segment the collaterals were considered adequate.

The mean delay from bleeding to endovascular treatment in the present series was eight days (range 1-30 days). The reason for the delay in the treatment in two cases (30 days) was due to misinterpretation of the findings of the first angiography before their admission in our hospital. All other patients were treated during the acute phase of the SAH (first to fourth post-bleeding day).

The aneurysms and proximal parent arteries were occluded using detachable coils or glue. Procedure-related complications and adverse events were recorded.

After the procedure, the patient was monitored in an intensive care unit.

Postprocedure CT scans were obtained in all patients within 48 hours. Seven patients underwent follow-up angiograms six months postoperatively. The final clinical outcome was assessed at the last clinical visit or phone interview by using the Glasgow Outcome Scale $(\mathrm{GOS})^{5}$.

\section{Results}

All patients presented with an acute intracranial hemorrhage (four patients with subarachnoid hemorrhage [SAH], five patients with SAH and intraventricular hemorrhage [IVH], and one patient SAH with intracerebellar hematoma), confirmed by CT. Hunt and Hess grading at admission was as follows: grade I, two patients; grade II, four patients; grade III, two patients; and grade IV, two patients. In four cases, the aneurysm was located on the left side, in six cases, on the right side. The location of the aneurysms was anterior medullary segment in three lateral medullary segment in three, telovelotonsillar segment in two, and cortical segment in two cases. Aneurysm size ranged from three to $15 \mathrm{~mm}$ in the longest axis.

All aneurysms were successfully treated on the first attempt. The endovascular treatment method was parent artery occlusion in all patients. Parent artery occlusion was performed with coils in eight cases (Figures 1 and 2), and with liquid embolic agent in two cases (cortical segment). In small distally located aneurysms (cortical segments) embolization with detachable coils would have been difficult and therefore we used liquid embolic agent. One of the lesions was embolized using Histoacryl (Auscelap, B. Braun) and one using Glubran 2 (GEM Srl). A $60 \%$ mixture of glue was used. To avoid distal penetration of the glue or reflux into the parent vessel, in all cases a balloon (Hyper- 

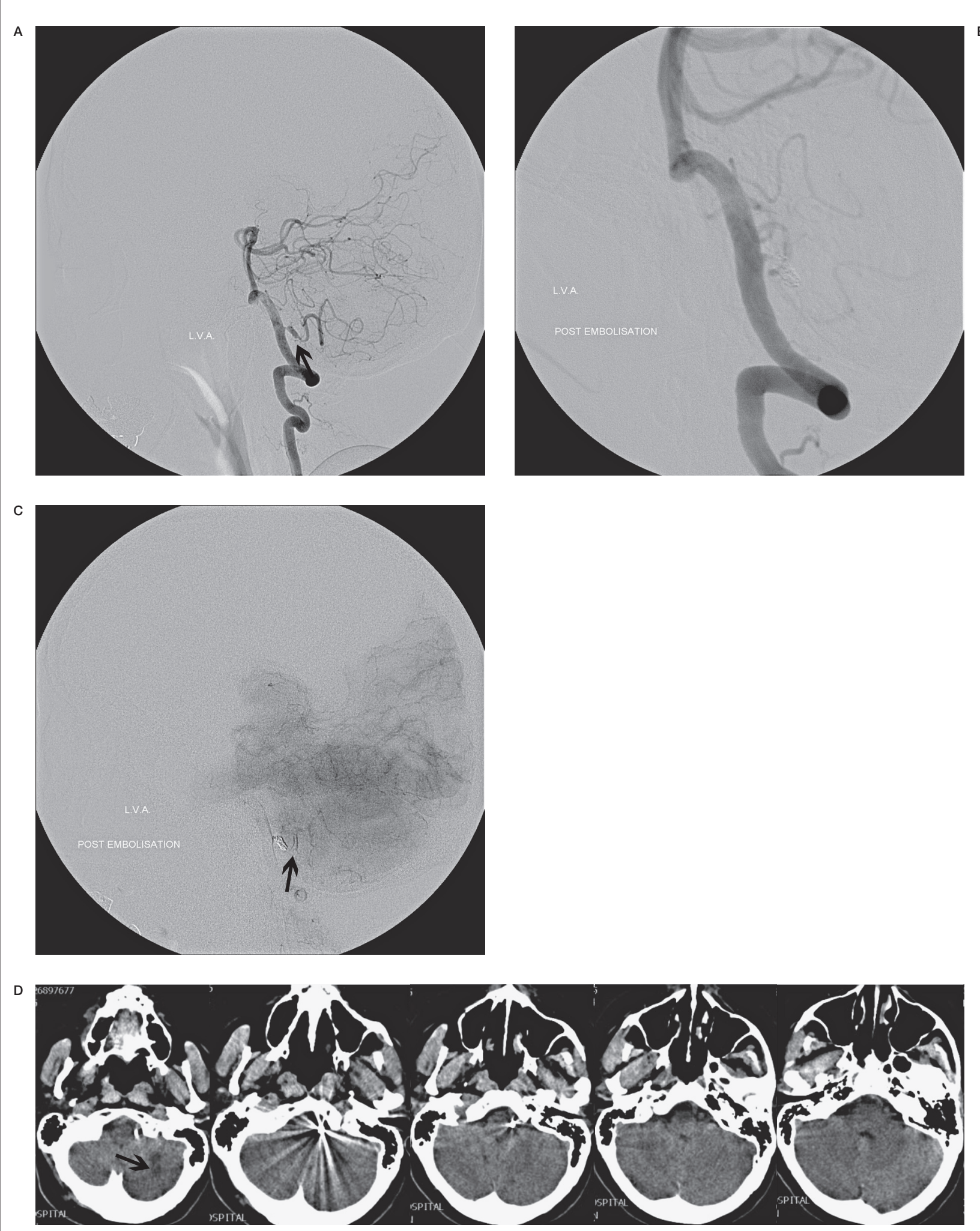

Figure 1 Images of a 58-year-old woman with SAH. A) Left vertebral artery injection, lateral view, shows a dissecting aneurysm arising from the anterior medullary segment of the left PICA (arrow). B) Post-embolization angiogram, shows complete occlusion of the left PICA. C) Post-embolization angiogram, lateral view, late phase, shows retrograde filling of the occluded PICA (arrows). D) Post-embolization CT images of the posterior fossa show a small infarct of the left cerebellar hemisphere. The patient remained neurologically intact. 
Form Occlusion Balloon System, ev3) was inflated across the origin of the PICA and the glue was injected slowly.

At the end of each procedure, occlusion of the aneurysm was confirmed by a selective angiogram. The retrograde filling of the distal segments of the occluded vessel was also evaluated (Figure 1C, 2D). There were no procedural complications.

Post-treatment CT scans within 48 hours obtained in all cases. In two cases (aneurysms of the anterior medullary and lateral medullary segments of PICA), asymptomatic, small, cerebellar infarcts were detected.

Follow-up angiographic studies (six months post-treatment) were performed in seven cases (one patient lost and two patients died) showed stable and complete occlusion in all cases. No retreatments were performed.

The patients were followed up clinically over a mean period of 12 months (range six to 28 months). The long-term clinical outcome on follow-up was assessed using the Glasgow outcome scale (GOS). The clinical outcome was good (GOS 5) in eight cases, whereas two patients with poor clinical condition at admission (Hunt \& Hess grade IV) died during their initial hospital stay ( $9^{\text {th }}$ and $17^{\text {th }}$ post-bleed day).

There were no rebleedings after the endovascular treatment or during the follow-up period.

\section{Discussion}

The PICA typically arises from the intracranial portion of the VA (80-95\% of cases). The PICA can be divided into five segments and two loops, based on its relationship with the medulla oblongata and the cerebellum. The segments are the following: anterior medullary, lateral medullary, tonsillomedullary, telovelotonsillar, and cortical $^{4}$. Dissecting aneurysm is diagnosed if an irregular fusiform dilatation with or without associated narrowing of the PICA demonstrated angiographically ${ }^{6}$. In the majority of cases the etiology of dissecting aneurysms is unknown ${ }^{7}$.

PICA dissecting aneurysms account for approximately 0.5 to $0.7 \%$ of all intracranial aneurysms 2. Subarachnoid hemorrhage is the most common initial clinical presentation (74\%) with ischemia accounting for the remaining cases ${ }^{8}$. Kanou et al. ${ }^{9}$ reported that dissecting aneurysms of the proximal PICA tend to cause infarctions and that peripheral dissections tend to lead to SAH. In our series all patients presented with subarachnoid hemorrhage with or without intraventricular hemorrhage, or intracerebellar hematoma.

Dissecting aneurysm of the PICA carries a $24 \%$ risk of rebleeding in the acute phase ${ }^{6}$, associated with a high mortality rate and early intervention is essential.

Table 1 Patient and aneurysm characteristics.

\begin{tabular}{|c|c|c|c|c|c|c|}
\hline & Age/sex & $\begin{array}{l}\text { Hunt \& } \\
\text { Hess } \\
\text { Grade }\end{array}$ & CT Findings & Location & Treatment & $\begin{array}{l}\text { Clinical } \\
\text { Outcome }\end{array}$ \\
\hline 1 & $\mathrm{~F} / 56$ & II & SAH & Left - cortical & Endovasular/glue & Excellent \\
\hline 2 & $\mathrm{~F} / 54$ & III & $\mathrm{SAH} / \mathrm{ICH}$ & Right - cortical & Endovascular/glue & Excellent \\
\hline 3 & $\mathrm{M} / 57$ & II & SAH/IVH & Right - telovelotonsillar & Endovascular/coils & Exellent \\
\hline 4 & $\mathrm{~F} / 54$ & I & SAH/IVH & Left - telovelotonsillar & Endovascular/coils & Excellent \\
\hline 5 & $\mathrm{~F} / 56$ & I & SAH & Left - lateral medullary & Endovascular/coils & Excellent \\
\hline 6 & $\mathrm{~F} / 55$ & IV & SAH & $\begin{array}{l}\text { Right - lateral } \\
\text { medullary }\end{array}$ & Endovascular/coils & Died \\
\hline 7 & $\mathrm{M} / 78$ & IV & $\mathrm{SAH} / \mathrm{IVH}$ & $\begin{array}{l}\text { Left - lateral } \\
\text { medullary }\end{array}$ & Endovascular/coils & Died \\
\hline 8 & $\mathrm{~F} / 59$ & II & SAH & $\begin{array}{l}\text { Right - anterior } \\
\text { medullary }\end{array}$ & Endovascular/coils & Excellent \\
\hline 9 & $\mathrm{M} / 56$ & III & SAH/IVH & $\begin{array}{l}\text { Right - anterior } \\
\text { medullary }\end{array}$ & Endovascular/coils & Excellent \\
\hline 10 & $\mathrm{~F} / 58$ & II & $\mathrm{SAH} / \mathrm{IVH}$ & $\begin{array}{l}\text { Right - anterior } \\
\text { medullary }\end{array}$ & Endovascular/coils & Excellent \\
\hline
\end{tabular}




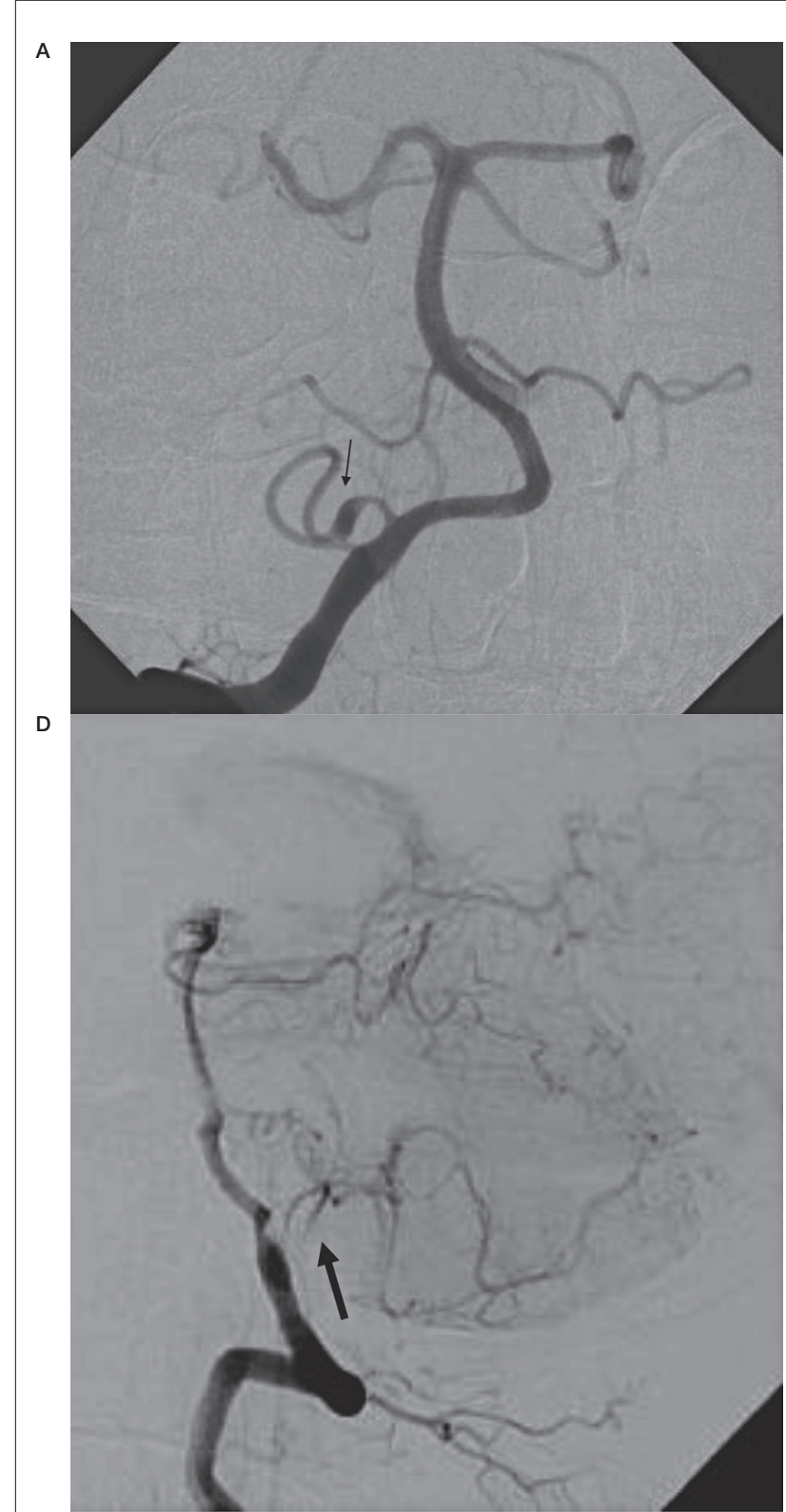

The management of PICA aneurysms that cause SAH is challenging and remains controversial. Most cases reported in the literature have been treated by surgical occlusion of the PICA or trapping of the dissected segment. They suggested that trapping of the involved segment with distal revascularization is the treatment of choice. More recently, successful endovascular treatment of dissecting PICA aneurysms has been reported ${ }^{6,710-12}$. The endovascular approach includes occlusion of the aneurysm and the parent artery at the dissected segment.

Parent artery occlusion of the PICA is potentially associated with two types of ischemic

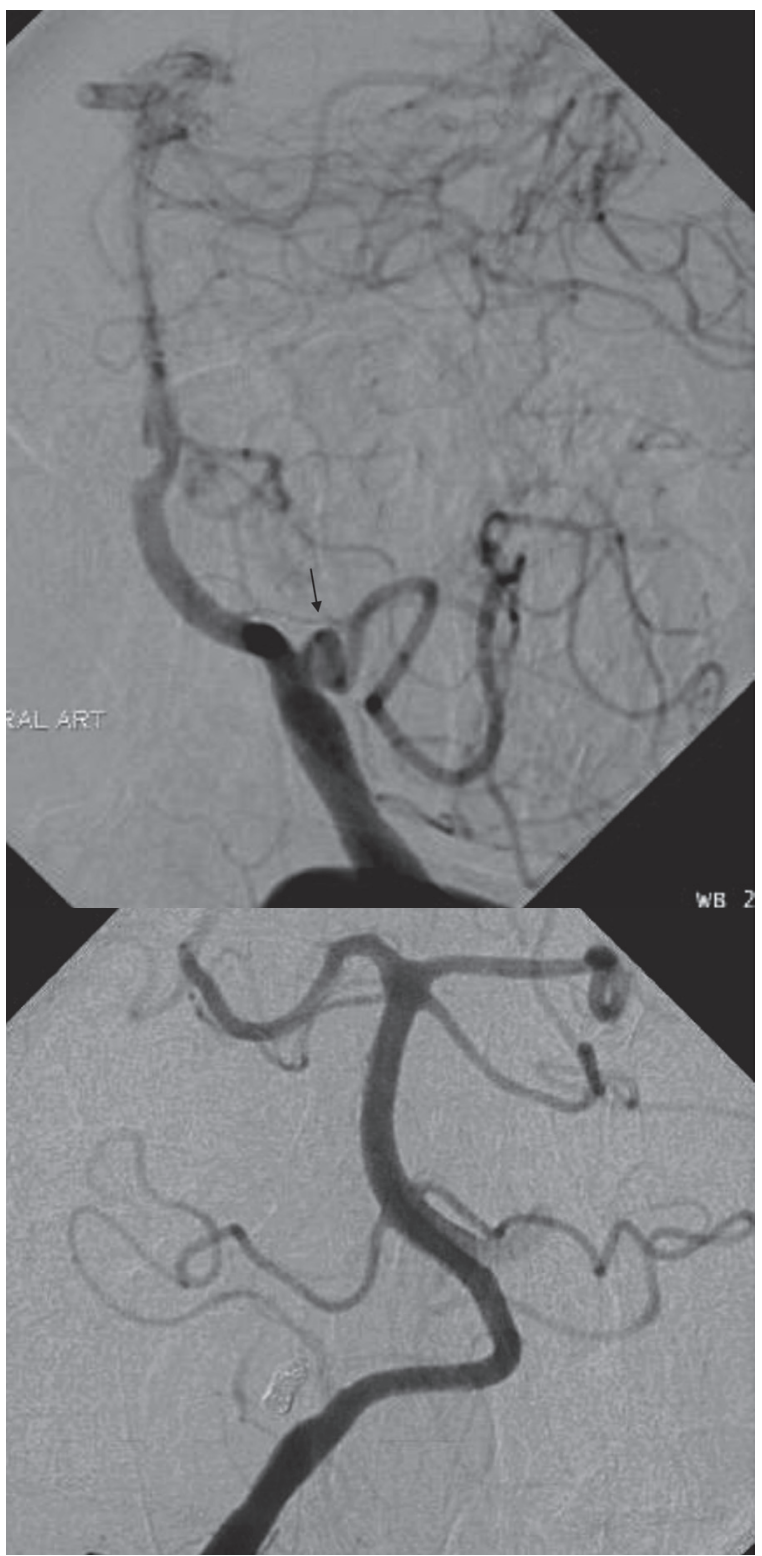

c

complication. First, brain stem ischemia due to occlusion of the perforating arteries supplying the brain stem which may originate from the six most proximal segments of the PICA and second cerebellar ischemia distal to the occlusion site.

In our group of patients there were three aneurysms located in the two more proximal segments of PICA (anterior and lateral medullary). To our knowledge, the present series is the largest subgroup of patients with dissected aneurysms in the most proximal segments of PICA. In all patients the aneurysm and the dissected segment occluded without neurological 


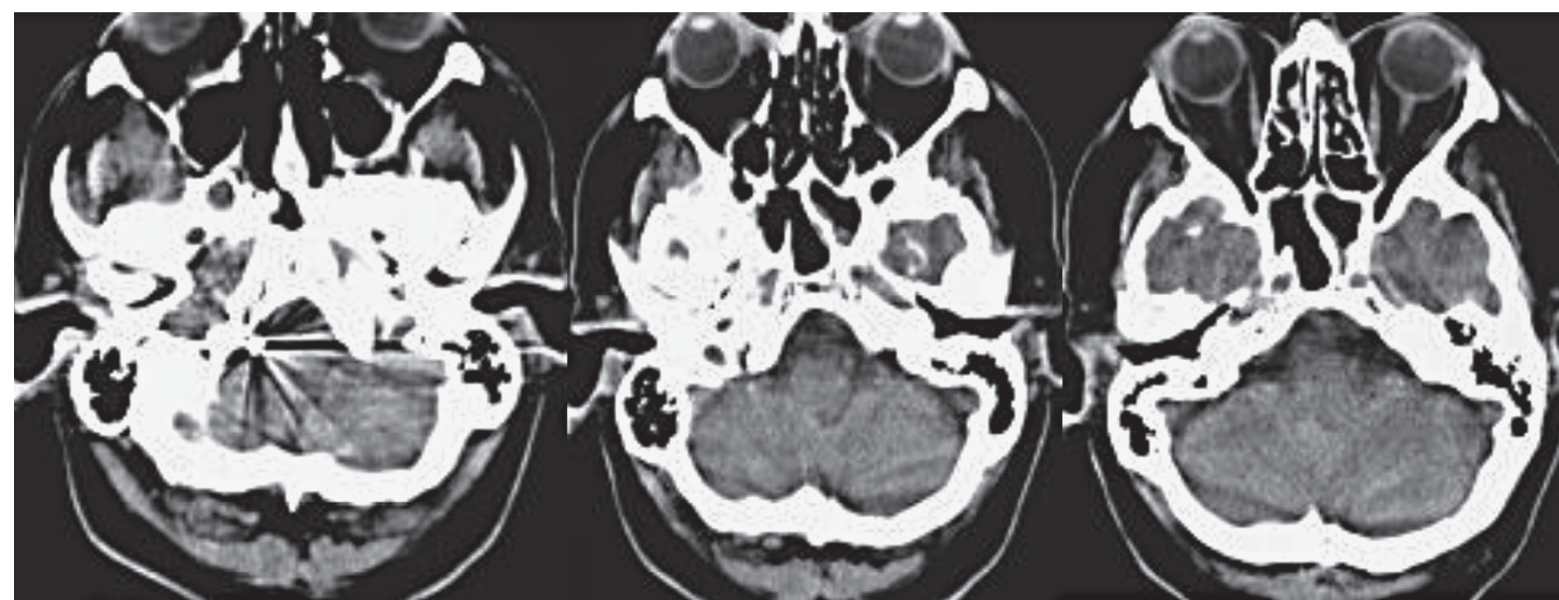

Figure 2 Images of a 67-year-old man with SAH. A,B) Right vertebral artery injection (AP \& lateral projections) shows a right PICA artery dissecting aneurysm arising from the lateral medullary segment (arrow). C,D) Post-embolization right vertebral injection (AP \& lateral projections). Note on the lateral projection the retrograde filling of the occluded vessel (arrow). E) Post-embolization serial CT images of the posterior cranial fossa with no additional lesions.

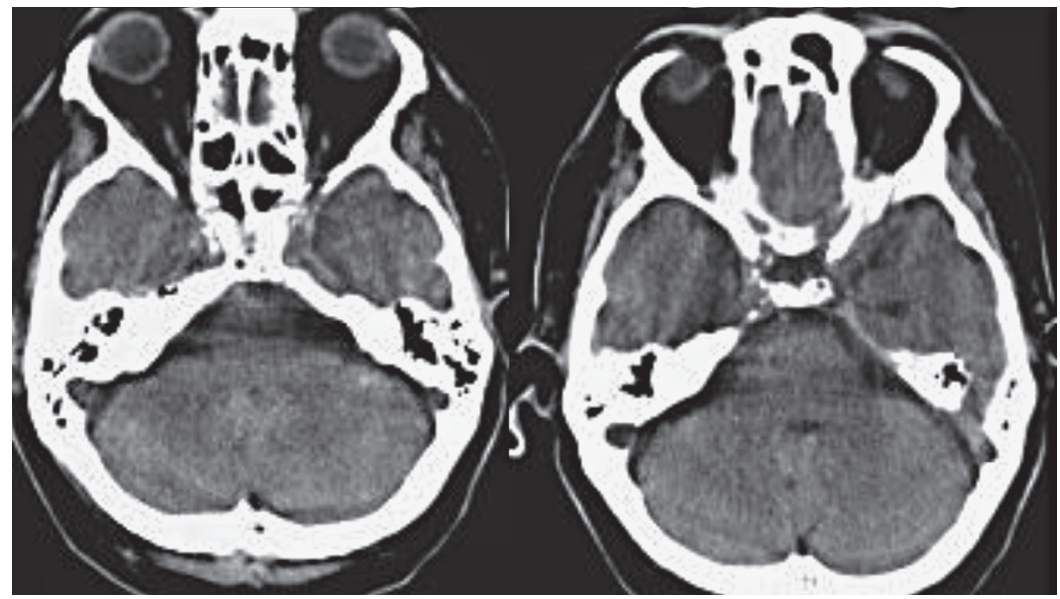

consequences. Additionally in the three most recent publications in the English literature ${ }^{6,7,10}$ which reported the results of endovascular treatment in dissecting PICA aneurysms, there were nine aneurysms located in the two more proximal segments. All aneurysms were treated endovascularly with parent artery occlusion with excellent neurological outcome. The risk of brain stem ischemia in cases of permanent occlusion at the first two segments is limited due to absence of normal perforating branches in aneurysmal dilatations of the dissected arterial segments ${ }^{13}$. Additionally, the risk of brain stem ischemia is also limited territories due to the numerous anastomoses of the perforating arteries forming a plexiform network on the medullary surface ${ }^{14}$. Furthermore, in both surgical and endovascular treatment the diseased segment is occluded and thus perforating vessels in that area are sacrificed.

On the other hand, occlusion of PICA distal to the telovelotonsillar segment generally does not result in brain stem injury. To assess the risks of PICA occlusion we performed detailed angiographic evaluation of the posterior fossa and the potential collateral supply from the contralateral PICA or ipsilateral anterior cerebellar artery and/or superior cerebellar artery 
was evaluated. Balloon test occlusion can be performed to accurately evaluate the patient's capability to tolerate permanent occlusion. In cases where collateral supply is insufficient, potentially there is the option of surgical bypass. On the other hand, if permanent PICA occlusion is followed by cerebellar infarct, it is usually of limited size, and patients usually tolerate it well 7,15 . PICA territory infarcts found in follow-up CT scan in two of our patients $(20 \%)$ after permanent PICA occlusion without neurological consequences. It is well known that MRI is more accurate in depicting ischemic lesions, especially in the posterior fossa, and therefore the incidence of post procedural cerebellar infarcts could be higher.

To conclude, according to our results, endovascular treatment of dissected PICA aneurysms with occlusion of both the dissecting aneurysm and the parent artery has excellent clinical outcome, although our group included the largest number of patients with dissections in the two most proximal segments. Despite the excellent clinical outcome careful preprocedural angiographic evaluation of the collateral circulation is necessary to avoid extensive cerebellar damage and edema especially in the setting of SAH.

\section{References}

1 Hudgins RJ, Day AL, Quisling RG, et al. Aneurysms of the posterior inferior cerebellar artery. A clinical and anatomical analysis. J Neurosurg. 1983; 58: 381-387.

2 Yamakawa H, Kaku Y, Yoshimura S, et al. Two cases of dissecting aneurysm of the distal posterior inferior cerebellar artery: possible involvement of segmental mediolytic arteriopathy in the pathogenesis. Clin Neurol Neurosurg. 2005; 107: 117-122.

3 Hunt WE, Hess RM. Surgical risk as related to time of intervention in the repair of intracranial aneurysms. $\mathbf{J}$ Neurosurg. 1968; 28: 14-20.

4 Lister JR, Rhoton AL Jr, Matsushima T, et al. Microsurgical anatomy of the posterior inferior cerebellar artery. Neurosurgery. 1982; 10: 170-199.

5 Jennett B, Bond M. Assessment of outcome after severe brain damage. Lancet. 1975, 1: 480-484.

6 Lim SM, Choi IS, Hum BA et al. Dissecting aneurysms of the distal segment of the posterior inferior cerebellar arteries: Clinical presentation and management. Am J Neuroradiol. 2010; 31: 1118-1122.

7 Maimon S, Saraf-Lavi E, Rappaport ZH, et al. Endovascular Treatment of Isolated Dissecting Aneurysm of the Posterior Inferior Cerebellar Artery Am J Neuroradiol. 2006; 27: v527-v532.

8 Tawk RG, Bendok BR, Qureshi AI, et al. Isolated dissections and dissecting aneurysms of the posterior inferior cerebellar artery: topic and literature review. Neurosurg Rev. 2002; 26: 180-187.

9 Kanou Y, Arita K, Kurisu K, et al. Dissecting aneurysm of the peripheral posterior inferior cerebellar artery. Acta Neurochir. 2000; 142: 1151-1156.

10 Isokangas JM, Siniluoto T, Tikkakoski T, et al. Endovascular treatment of peripheral aneurysms of the posterior inferior cerebellar artery. Am J Neuroradiol. 2008; 29: 1783-1788.

11 Tikkakoski T, Leinonen S, Siniluoto T, et al. Isolated dissecting aneurysm of the left posterior inferior cerebellar artery: endovascular treatment with a Guglielmi detachable coil. Am J Neuroradiol. 1997; 18: 936-938.

12 Mukonoweshuro W, Laitt RD, Hughes DG. Endovascular treatment of PICA aneurysms. Neuroradiology. 2003; 45: 188-192.

13 Dinichert A, Rufenacht DA, Tribolet N. Dissecting aneurysms of the posterior inferior cerebellar artery: report of four cases and review of the literature. J Clin Neurosci. 2000; 7: 515-120.
14 Duvernoy HM, ed. Human Brain Stem Vessels. Berlin: Springer; 1999.

15 Nussbaum ES, Madison MT, Myers ME, et al. Dissecting aneurysms of the posterior inferior cerebellar artery: retrospective evaluation of management and extended follow-up review in 6 patients. J Neurosurg. 2008; 109: 23-27.

16 Lewis SB, Chang DJ, Peace DA, et al. Distal posterior inferior cerebellar artery aneurysms: clinical features and management. J Neurosurg. 2002; 97: 756-766.

17 Horowitz M, Kopitnik T, Landreneau F, et al. Posteroinferior cerebellar artery aneurysms: surgical results for 38 patients. Neurosurgery. 1998; 43: 1026-1032.
Dr Ioannis Ioannidis "Hygeia" Hospital Erythrou Stavrou 4, Marousi Athens, 15123, Greece E-mail: iioann@hotmail.com 